\title{
Textural measures for estimating oil palm age
}

\begin{abstract}
In oil palm management, age is one of the yield determinant factors. The conventional field investigations are often exhaustive and costly methods when implemented on a large scale. Despite much attention to classify individual oil palm ages by using various remote sensing images, none of the studies depicted satisfying overall accuracies. The overall aim of this study was to optimize window size and number of texture measurements for oil palm ages classification. The study was conducted in a commercial oil palm plantation comprised of palms of multiple ages planted from 1991 to 2008. Three Satellite Pour l'Observation de la Terre (SPOT)-5 multispectral images, acquired on 12 April 2012, 4 April 2013, and 14 April 2014, were evaluated. The individual ages were successfully classified with accuracy ranging from $59 \%$ to $97 \%$, with an average overall accuracy of $84 \%$. The results illustrated that the largest window size related to the smallest oil palm planting block in the study area, $390 \mathrm{~m} \times$ $390 \mathrm{~m}$ on-the-ground window size, and seven combination of texture measurements, resulted in the highest classification overall accuracy. The utilization of texture measurements produced synergistic effects able to discriminate the oil palm age, with mean, entropy, homogeneity, and angular second moment as among the significant textures.
\end{abstract}

Keyword : Oil palm management; Remote sensing images; Oil palm 